\title{
Indicadores quantitativos da biologia reprodutiva de fêmeas de piau-vermelho no Rio Paraíba do Sul
}

\author{
Ana Paula Ribeiro Costa ${ }^{(1)}$, Dalcio Ricardo de Andrade ${ }^{(1)}$, Manuel Vazquez Vidal Junior $^{(1)}$ e Guilherme Souza(1)
}

(1)Universidade Estadual do Norte Fluminense, Centro de Ciências e Tecnologias Agropecuárias, Laboratório de Zootecnia e Nutrição Animal, Avenida Alberto Lamego № 2.000, Parque Califórnia, CEP 28015-130 Campos dos Goytacazes, RJ. E-mail: dalcio@uenf.br

\begin{abstract}
Resumo - O objetivo deste trabalho foi avaliar a dinâmica reprodutiva de fêmeas de piau-vermelho (Leporinus copelandii) na bacia do baixo Rio Paraíba do Sul. Os parâmetros foram determinados nos diferentes estádios do ciclo reprodutivo anual de 143 fêmeas obtidas mediante capturas mensais. Houve correlação positiva entre relação gonadossomática e fator de condição total, e correlação negativa entre relação gonadossomática e índice de gordura celomática. O fator de condição total e o fator somático apresentaram a mesma tendência, e a diferença entre os dois indicou o início do período reprodutivo. O índice hepatossomático apresentou correlação negativa com gordura celomática e correlação positiva com o índice de repleção estomacal. O índice de gordura celomática apresentou correlação positiva com os fatores de condição total e somático. O índice médio de gordura celomática foi máximo no estádio de maturação inicial, sugerindo a utilização destas reservas durante a maturação gonadal e/ou migração reprodutiva. Houve correlação negativa entre índice de gordura celomática e índice de repleção estomacal. Apesar de não ter ocorrido interrupção na alimentação, as fêmeas apresentaramse em melhores condições alimentares após o período reprodutivo.
\end{abstract}

Termos para indexação: Leporinus copelandii, reprodução de peixes, índice gonadossomático, Anostomidae.

\section{Indicators on the reproductive biology of female red-piau in Paraíba do Sul River}

\begin{abstract}
The objective of this work was to evaluate the reproductive dynamics of red-piau females (Leporinus copelandii) in the lower basin of Paraíba do Sul River. Variables were determined in different stages of annual reproductive cycle of the 143 monthly caught females. There was a significant correlation between gonadosomatic relationship and total condition factor, and a negative correlation between gonadosomatic relationship and abdominal fat index. Total and somatic condition factor presented the same tendency, and their difference indicated the beginning of the reproductive period. Hepatosomatic index presented negative correlation with abdominal fat index, and positive correlation with repletion index. Abdominal fat index presented positive correlation with total and somatic condition factor. The mean abdomen fat index was maximum in the initial maturation stage suggesting the use of fat reservations during the ovaries maturation and/or reproductive migration. There was a negative correlation between abdominal fat index and the repletion index. Despite the fact that the fish did not interrupt its alimentary activity, the females showed better alimentary conditions after the reproductive period.

Index terms: Leporinus copelandii, fish reproduction, gonadossomatic index, Anostomidae.
\end{abstract}

\section{Introdução}

Ao longo do ciclo reprodutivo anual dos peixes, modificações marcantes podem ser observadas em suas gônadas (Nikolski, 1963), principalmente no que diz respeito ao seu peso, decorrente, em grande parte, do acúmulo de material de reserva nos ovócitos em maturação (Wooton, 1995; Vazzoler, 1996). Como indicativo do estado funcional dos ovários, freqüentemente tem-se reportado ao índice gonadossomático, uma vez que este expressa a porcentagem que as gônadas representam do peso total ou do peso corporal dos indivíduos (Vazzoler, 1996).
A fonte energética e os nutrientes necessários para o processo de maturação ovocitária e desencadeamento da reprodução nos peixes são obtidos a partir do alimento ingerido ou de reservas energéticas depositadas em diferentes partes do organismo (Vazzoler, 1996). Portanto, ao conhecer a curva de maturação da espécie, pode-se correlacioná-la ao estado nutricional do indivíduo e à capacidade de mobilização destas reservas (Bazzoli et al., 1996).

O objetivo deste trabalho foi determinar os índices quantitativos relativos aos estádios do ciclo reprodutivo, da condição nutricional e das reservas energéticas, e suas correlações com o desenvolvimento gonadal de 
fêmeas de Leporinus copelandii na região do baixo Rio Paraíba do Sul, entre os municípios de Itaocara e Campos dos Goytacazes, RJ.

\section{Material e Métodos}

Foram capturadas 143 fêmeas de L. copelandii na bacia do baixo Rio Paraíba do Sul, entre os municípios de Itaocara e Campos dos Goytacazes, RJ (21041'15"S; $42^{\circ} 3^{\prime} 45^{\prime \prime} \mathrm{W}$ ), em amostragens mensais, compreendidas entre fevereiro de 1998 e março de 1999, utilizandose redes de espera (malhas 3 a $6 \mathrm{~cm}$ entre nós). Os animais foram transportados para o laboratório em caixas de isopor contendo gelo e manuseados logo em seguida.

Na obtenção dos dados biométricos, os exemplares foram medidos e pesados. Após incisão ventral, os peixes foram dissecados, e ovários e vísceras foram retirados e pesados. De cada exemplar, foi obtido o comprimento padrão (CP), peso total (PT), peso das gônadas (PG), peso do fígado (PF), peso do estômago (PE) e peso da gordura celomática (PGC).

Para analisar quantitativamente a atividade reprodutiva, foram calculados os seguintes índices: relação gonadossomática [RGS = $(\mathrm{PG} / \mathrm{PT}) \times 100]$, segundo Vazzoler (1996) e índice hepatossomático [IHS $=(\mathrm{PF} / \mathrm{PT}) \times 100]$, índice de gordura celomática [IGC $=(\mathrm{PGC} / \mathrm{PT}) \times 100]$ e índice de repleção do estômago [IRE $=(\mathrm{PE} / \mathrm{PT}) \times 100]$, segundo Andrade (1990).

Os estádios de repleção estomacal (ERE) foram determinados por meio de avaliação visual quantitativa do conteúdo estomacal dos exemplares coletados, classificando-os de acordo com escala descrita em Santos (1986), em que, estádio I (ERE I): estômago vazio; estádio II (ERE II): estômago semi-cheio, e estádio III (ERE III): estômago cheio.

$\mathrm{O}$ fator de condição corrigido $(\mathrm{K})$ foi estimado para cada indivíduo pela expressão: $\mathrm{K} 1=\mathrm{PT} / \mathrm{CP}^{\mathrm{b}}$, e $\mathrm{K} 2=(\mathrm{PT}-\mathrm{PG}) / \mathrm{CP}^{\mathrm{b}}$, em que $\mathrm{b}$ representa o coeficiente angular da regressão entre peso total e comprimento padrão (constante relacionada com o tipo de crescimento anual da espécie), estabelecida pela equação $\mathrm{PT}=\mathrm{a} \times \mathrm{CP}^{\mathrm{b}}$ (Vazzoler, 1982, 1996; Barbieri et al., 1982).

A relação peso total/comprimento padrão foi determinada para verificar o tipo de crescimento da espécie. Os valores foram lançados em gráfico de dispersão, considerando-se como variável dependente o peso total e independente, o comprimento padrão.
O período reprodutivo foi estabelecido pela distribuição dos valores médios mensais da relação gonadossomática (RGS) e da freqüência relativa dos estádios do ciclo reprodutivo identificados histologicamente e definidos como repouso, maturação inicial, maturação intermediária, maturação avançada, maduro e desovado, segundo método utilizado por Vazzoler (1982, 1996).

Foram realizadas análises estatísticas descritivas das variáveis relação gonadossomática, índice hepatossomático, índice de gordura celomática, índice de repleção estomacal e fator de condição $(\mathrm{K})$ nos estádios do ciclo reprodutivo. A associação entre as variáveis foi analisada por correlação de Pearson (r) (Pimentel-Gomes, 1990).

\section{Resultados e Discussão}

A dispersão dos pontos de comprimento padrão e peso total seguiu a tendência de curva potencial de forma $\mathrm{Y}=\mathrm{aX}$, representada pela equação $\mathrm{PT}=-1,4373 \mathrm{CP}^{2,7913}$; $\mathrm{r}^{2}=0,96 ; \mathrm{n}=143$.

A relação peso total/comprimento padrão mede a variação do peso esperado em função do comprimento e vice-versa, e indica a condição do peixe pelo acúmulo de gordura, bem-estar geral e desenvolvimento gonadal (Le Cren, 1951).

O valor do coeficiente angular de regressão entre peso e comprimento varia de 2,5 a 4,0 (Vazzoler, 1996). Em fêmeas de $L$. copelandii, o valor de b foi igual a $2,79\left(\chi^{2}>0,01\right)$, valor próximo de 3,0, que, segundo Vazzoler (1996), é característico de espécies que apresentam crescimento isométrico.

Entre as fêmeas de L. copelandii coletadas, o menor exemplar apresentou comprimento padrão de $19,1 \mathrm{~cm}$ e o maior de 35,3 cm, com ovários em estádio de maturação avançada e desovado, respectivamente.

Ao longo do período de coleta, foi observado um patamar representado pelos valores médios mensais de relação gonadossomática no período de fevereiro a julho/1998. A partir do mês de agosto/1998, ocorreu incremento nos valores de RGS, o qual atingiu valor mé-

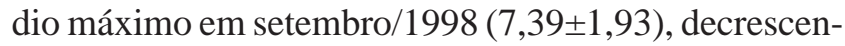
do nos meses subseqüentes (Tabela 1).

A análise do ciclo reprodutivo anual, realizada a partir dos valores médios mensais de RGS, possibilitou inferir que o período de fevereiro a julho de 1998 está associado, provavelmente, a uma fase de recuperação gonadal do ciclo reprodutivo anterior, e sua reorganiza- 
ção para o subseqüente. A maturação foi iniciada a partir de agosto, atingindo o pico em setembro/1998, quando os indivíduos foram considerados aptos à reprodução.

Pela análise descritiva da variável RGS, por estádio do ciclo reprodutivo de $L$. copelandii, foi observado que os valores médios aumentaram do estádio de repouso $(0,39 \pm 0,03)$ até alcançar valor máximo no subestádio de maturação avançada $(5,55 \pm 1,17)$, para, em seguida, decrescer no estádio de desova $(1,58 \pm 0,31)$. Nos estádios de repouso e maturação inicial, os valores obtidos de RGS apresentaram sobreposição dos respectivos intervalos de confiança (Tabela 2).

Diniz (1997) observou que fêmeas de Leporinus striatus atingiram valor máximo de RGS igual a 7,33 $\pm 5,08$ na represa de Camargos, MG. Porém fêmeas de Leporinus piau (Tavares \& Godinho, 1994) atingiram valor médio de relação gonadossomática igual a 23,3 $\pm 3,2$ na represa de Três Marias, MG. Lopes et al. (1999), trabalhando com outro anostomídeo (Leporinus friderici), em ambientes lênticos e lóticos, encontraram menores valores de fator de condição em peixes no ambiente lótico, próximo ao local da desova, além de variação, até mesmo, de acordo com a época reprodutiva.
Entretanto, em ambientes lênticos, a condição corporal variou de acordo com o local de desova, e a condição corporal nesse ambiente foi maior. O autor associa essa discrepância a diferenças limnológicas (temperatura, fluxo de água, pluviosidade) e também a disponibilidade de alimento. Soma-se ainda a estas informações, o maior dispêndio energético necessário nas migrações reprodutivas, no caso do ambiente lótico.

As gônadas dos peixes de ciclo reprodutivo anual são, de um modo geral, submetidas à variações marcantes de volume e peso devido ao avanço do processo de maturação ovocitária. Esta variação, comumente expressa pela relação gonadossomática, tem sido utilizada como indicador eficiente do estado funcional dos ovários em Leporinus friderici (Barbieri \& Santos, 1988), Schizodon knerii (Ferreira \& Godinho, 1990), Curimatella lepidura (Andrade, 1990), Leporinus piau (Tavares \& Godinho, 1994), Serrasalmus brandtii (Teles \& Godinho, 1997). Entretanto, a dinâmica deste coeficiente está intimamente associada ao tipo de desova da espécie (Nikolsky, 1963). No caso do Leporinus copelandii a histologia dos ovários indicou que a desova deste peixe é total.

Tabela 1. Valores mensais (média \pm intervalo de confiança) da relação gonadossomática (RGS), do fator de condição total (K1), fator somático (K2) e variação entre ambos (Dk), e índice de gordura celomática (IGC), para Leporinus copelandii, no período de fevereiro de 1998 a março de 1999.

\begin{tabular}{|c|c|c|c|c|c|c|}
\hline Meses & RGS & K1 & K2 & IGC & $\mathrm{k}$ & $\mathrm{n}$ \\
\hline Fevereiro/98 & $0,36 \pm 0,05$ & $0,0351 \pm 0,0030$ & $0,0350 \pm 0,0030$ & $4,38 \pm 3,06$ & 0,01 & 7 \\
\hline Março & $0,35 \pm 0,05$ & $0,0370 \pm 0,0022$ & $0,0368 \pm 0,0022$ & $5,86 \pm 1,37$ & 0,02 & 7 \\
\hline Abril & 0,24 & 0,0372 & 0,0371 & 3,48 & 0,01 & 1 \\
\hline Maio & $0,37 \pm 0,07$ & $0,0374 \pm 0,0015$ & $0,0373 \pm 0,0015$ & $5,20 \pm 1,37$ & 0,01 & 8 \\
\hline Junho & $0,38 \pm 0,11$ & $0,0368 \pm 0,0012$ & $0,0366 \pm 0,0012$ & $4,13 \pm 0,85$ & 0,02 & 15 \\
\hline Julho & $0,47 \pm 0,17$ & $0,0355 \pm 0,0014$ & $0,0353 \pm 0,0014$ & $2,93 \pm 0,99$ & 0,02 & 9 \\
\hline Agosto & $2,08 \pm 0,74$ & $0,0364 \pm 0,0010$ & $0,0356 \pm 0,0009$ & $2,05 \pm 0,74$ & 0,08 & 13 \\
\hline Setembro & $7,39 \pm 1,93$ & $0,0388 \pm 0,0029$ & $0,0359 \pm 0,0027$ & $0,29 \pm 0,29$ & 0,29 & 6 \\
\hline Outubro & $5,58 \pm 2,77$ & $0,0392 \pm 0,0023$ & $0,0370 \pm 0,0025$ & $1,28 \pm 1,09$ & 0,22 & 10 \\
\hline Novembro & $1,83 \pm 2,13$ & $0,0386 \pm 0,0050$ & $0,0379 \pm 0,0045$ & $2,52 \pm 1,07$ & 0,07 & 3 \\
\hline Dezembro & $1,45 \pm 0,53$ & $0,0361 \pm 0,0011$ & $0,0356 \pm 0,0011$ & $1,51 \pm 0,67$ & 0,05 & 13 \\
\hline Janeiro/99 & $2,24 \pm 0,55$ & $0,0370 \pm 0,0012$ & $0,0361 \pm 0,0012$ & $1,46 \pm 0,64$ & 0,09 & 30 \\
\hline Fevereiro & $2,03 \pm 0,93$ & $0,0357 \pm 0,0011$ & $0,0350 \pm 0,0009$ & $1,23 \pm 0,68$ & 0,07 & 14 \\
\hline Março & $0,51 \pm 0,10$ & $0,0363 \pm 0,0013$ & $0,0361 \pm 0,0013$ & $2,22 \pm 1,02$ & 0,02 & 7 \\
\hline
\end{tabular}

Tabela 2. Valores médios e intervalo de confiança $(\alpha=0,05)$ da relação gonadossomática (RGS), índice hepatossomático (IHS), índice de gordura celomática (IGC), índice de repleção estomacal (IRE), fator de condição total (K1) e fator somático (K2), por estádios do ciclo reprodutivo de fêmeas de Leporinus copelandii, durante o período de fevereiro/1998 a março/1999.

\begin{tabular}{lcccccc}
\hline Estádios & RGS & IHS & IGC & IRE & K1 & K2 \\
\hline Repouso & $0,39 \pm 0,03$ & $0,83 \pm 0,10$ & $3,55 \pm 0,59$ & $1,99 \pm 0,42$ & $0,0363 \pm 0,0006$ & $0,0361 \pm 0,0006$ \\
Maturação inicial & $0,46 \pm 0,09$ & $0,88 \pm 0,19$ & $3,82 \pm 1,18$ & $1,93 \pm 0,99$ & $0,0373 \pm 0,0013$ & $0,0372 \pm 0,0013$ \\
Maturação intermediária & $2,57 \pm 0,48$ & $1,09 \pm 0,11$ & $1,53 \pm 0,54$ & $2,36 \pm 0,76$ & $0,0368 \pm 0,0011$ & $0,0358 \pm 0,0011$ \\
Maturação avançada & $5,55 \pm 1,17$ & $0,96 \pm 0,14$ & $1,00 \pm 0,48$ & $2,13 \pm 0,98$ & $0,0381 \pm 0,0012$ & $0,0359 \pm 0,0010$ \\
Desovado & $1,58 \pm 0,31$ & $1,17 \pm 0,12$ & $1,32 \pm 0,50$ & $3,95 \pm 1,00$ & $0,0361 \pm 0,0012$ & $0,0355 \pm 0,0012$ \\
\hline
\end{tabular}


Valores mensais do fator de condição total e somático em $L$. copelandii (média \pm intervalo de confiança) durante o período de fevereiro/1998 a julho/1998 praticamente apresentaram a mesma tendência (Tabela 1). A partir do mês de agosto/1998, a diferença entre estas variáveis começou a aumentar, apresentando em setembro/1998 maior valor $(\mathrm{Dk}=0,29)$. O peso das gônadas influenciou diretamente o cálculo dos fatores de condição, K1 e K2, o que pode ser observado pela diferença entre eles, expressando assim, de modo relativo, a possível parcela das reservas energéticas transferidas para as gônadas no processo de maturação ovocitária. Observação semelhante à descrita por Vazzoler (1996), em relação a algumas espécies de peixes teleósteos.

O fator de condição é um indicador quantitativo do grau de bem-estar momentâneo do peixe, variável durante o ciclo de maturidade sexual do indivíduo, portanto, pode ser influenciado, entre outros fatores, pela variação no peso das gônadas, estômago e gordura celomática (Agostinho et al., 1990; Vazzoler, 1996). Em Rhinelepis aspera, a influência das gônadas não foi significativa, permitindo a utilização do peso total para o cálculo do fator de condição (Agostinho et al., 1990). Neste estudo, com a elevação do valor do Dk, foi possível identificar o início do período reprodutivo de L. copelandii. Resultado semelhante ao obtido em relação à Curimatella lepidura (Andrade, 1990).

Os valores de K1 e K2 não apresentaram a mesma tendência de variação ao longo dos estádios do ciclo reprodutivo (Tabela 2). Ambos aumentaram do estádio de repouso para o subestádio de maturação inicial, seguidos por diminuição no estádio de maturação intermediária. A partir de então, pôde-se observar a influência do peso das gônadas na obtenção de K, pois, enquanto os valores de K2 continuaram regredindo, até atingir o mínimo no estádio desovado, o K1, no qual se inclui o peso gonadal, elevou-se no $2 \mathrm{C}$, e, a partir daí, diminuiu no estádio 4.

De acordo com estes dados, K2 expressou melhor a condição corporal das fêmeas de $L$. copelandii durante os estádios do ciclo reprodutivo. Portanto, pode-se inferir que os indivíduos apresentaram melhores condições corporais no início do período reprodutivo (maturação inicial). Segundo Agostinho et al. (1990), a reposição das reservas corporais é indicada pelo incremento no fator de condição.

A redução nos valores do fator de condição na época de reprodução foi registrada em outras espécies por vários autores (Barbieri et al., 1982; Bazzoli, 1985;
Agostinho et al., 1990; Lopes et al., 1999). Barbieri et al. (1992) estudando Astyanax scabripinnis paranae, espécie de desova parcelada, observaram que os maiores valores do fator de condição ocorreram durante o período reprodutivo, sendo um bom indicador do período de desova desta espécie.

Vazzoler (1996) descreve que espécies que apresentam migrações reprodutivas, como é o caso do piauvermelho, além de transferirem reservas para as gônadas, consomem uma parcela das reservas energéticas somáticas neste processo, refletido no fator de condição somático (K2). Assim, peixes migradores, em função do gasto energético para atingir o local de desova, consomem energia e diminuem seu fator de condição conforme foi observado neste estudo.

A condição de bem-estar total do peixe (K1) foi significativamente correlacionada com o estádio do ciclo reprodutivo (RGS) $(\mathrm{r}=0,26 ; \mathrm{P}<0,001 ; \mathrm{n}=143)$, demonstrando ser um bom indicador do período reprodutivo.

Os valores do índice hepatossomático (IHS), para L. copelandii, não apresentaram correlação significativa com a relação gonadossomática (RGS) $(r=0,06$; $\mathrm{T}=0,7523 ; \mathrm{P}=0,23 ; \mathrm{n}=143$ ).

Os valores do IHS por estádio do ciclo reprodutivo elevaram-se do estádio de repouso até o subestádio de maturação intermediária, e apresentaram queda no subestádio de maturação avançada, aumentando em seguida, no estádio desovado, quando atingiram valor médio $(1,17 \pm 0,12 ; \mathrm{n}=19)$ (Tabela 2).

Embora não se tenha avaliado qualitativa nem quantitativamente a atividade do fígado durante a maturação gonadal, a diminuição do IHS do subestádio de maturação intermediária para a avançada pode ter sido atribuída à possível participação do fígado na síntese e secreção de substâncias hepáticas para formação do vitelo exógeno no processo de maturação ovocitária (Bazzoli et al., 1998).

Entretanto, Bazzoli (1985) relata que, em A. lacustris, o IHS, após o estádio de maturação avançada, continuou diminuindo ao longo do período de desova. Comportamento semelhante foi encontrado em $R$. aspera por Agostinho et al. (1990), os quais sugerem que a maturação ovocitária e o processo de reprodução levam à depleção as reservas orgânicas da espécie.

Andrade (1990), em estudo com C. lepidura, quando eliminou a influência dos ovários do cálculo do IHS, pôde observar a participação do fígado no processo vitelogênico. 
O índice hepatossomático apresentou correlação negativa significativa com o índice de gordura celomática $(\mathrm{r}=-0,30 ; \mathrm{P}<0,001 ; \mathrm{n}=143)$, demonstrando uma possível hipertrofia hepática para o catabolismo das reservas lipídicas, que seriam, então, participantes do processo de vitelogenese.

O índice de repleção estomacal (IRE), por sua natureza quantitativa, fornece informações mais precisas quanto à época em que os indivíduos se apresentam em melhores condições alimentares (Barbieri, 1981). Neste estudo, quanto ao estádio de repouso e período de maturação das gônadas, os valores de IRE se mantiveram estáveis, para, posteriormente à desova, atingirem seu valor máximo $(3.95 \pm 1,00)$ (Tabela 2$)$. Com esta observação, pode-se sugerir que fêmeas de L. copelandii ingerem menor quantidade de alimentos quando o fator de condição corporal é mais elevado, coincidindo com a época da migração reprodutiva. Um fator importante é que a maturação gonadal acaba limitando o espaço peritonial, exercendo uma pressão mecânica sobre o trato gastrointestinal, reduzindo, portanto, a ingestão de alimentos. Após a desova, maiores valores de IRE indicam que esses animais retomam a ingestão normal de alimentos.

A análise das freqüências absoluta $(\mathrm{Fa})$ e relativa $(\mathrm{Fr})$ dos estádios de repleção estomacal I $(\mathrm{Fa}=47 ; \mathrm{Fr}=32,87 \%)$, II ( $\mathrm{Fa}=28 ; \mathrm{Fr}=19,59 \%)$ e III ( $\mathrm{Fa}=68 ; \mathrm{Fr}=47,55 \%)$ mostrou que a maioria dos exemplares capturados apresentavam estômago cheio (ERE III), fato observado em todos os estádios do ciclo reprodutivo (Tabela 3). Estas duas informações permitem inferir que o $L$. copelandii não interrompeu sua atividade alimentar ao longo do período de amostragem, embora a capacidade de ingestão de alimento tenha sido reduzida pela compressão mecânica dos ovários sobre o trato digestório no período reprodutivo. Isto explica o menor índice de repleção e do fator de condição no período reprodutivo.

Tabela 3. Distribuição da freqüência absoluta $(\mathrm{Fa})$ e relativa (Fr) dos estádios de repleção estomacal (ERE) por estádio do ciclo reprodutivo de fêmeas de Leporinus copelandii, no período de fevereiro de 1998 a março de 1999.

\begin{tabular}{lcccccccc}
\hline Estádios & \multicolumn{2}{c}{ ERE I } & & \multicolumn{2}{c}{ ERE II } & & \multicolumn{2}{c}{ ERE III } \\
\cline { 2 - 3 } & $(\mathrm{Fa})$ & $(\mathrm{Fr} \%)$ & & $(\mathrm{Fa})$ & $(\mathrm{Fr} \%)$ & & $(\mathrm{Fa})$ & $(\mathrm{Fr} \%)$ \\
\hline Inicial & 23 & 43,4 & & 9 & 17,0 & & 21 & 39,6 \\
Maturação inicial & 9 & 53,0 & & 4 & 23,5 & & 4 & 23,5 \\
Maturação intermediária & 9 & 30,0 & & 6 & 20,0 & & 15 & 50,0 \\
Maturação avançada & 5 & 20,8 & & 9 & 37,5 & & 10 & 41,7 \\
Desovado & 1 & 5,3 & & $-{ }^{(1)}$ & - & & 18 & 94,7 \\
\hline
\end{tabular}

(1)Evento não observado.
O RGS não apresentou correlação significativa com IRE ( $r=-0,13 ; T=-1,5936 ; P=0,06 ; n=143)$. Esta associação negativa, embora não-significativa no presente estudo, entre o período reprodutivo (RGS) e a atividade alimentar (IRE), segundo Tavares (1986) e Andrade (1990) deve-se à compressão mecânica exercida pelas gônadas nos órgãos dentro da cavidade celomática, o que diminui a capacidade de repleção estomacal. Houve correlação significativa entre a atividade alimentar e o peso do fígado ao longo do período reprodutivo $(r=0,39 ; \mathrm{P}<0,001 ; \mathrm{n}=143)$. Isso provavelmente se deve a uma maior atividade hepática para metabolizar os alimentos ingeridos principalmente no tocante ao aspecto de produção de vitelogenina pelo fígado.

Os maiores valores médios do índice de gordura celomática foram observados no estádio de repouso $(3,55 \pm 0,59)$ e maturação inicial $(3,82 \pm 1,18)$, decrescendo em seguida nos subestádios de maturação intermediária, maturação avançada e desovado (Tabela 2). Esta deposição de gordura sugere uma preparação do indivíduo, por meio do acúmulo de reservas energéticas, para o período reprodutivo, preparando, assim, o peixe para o processo migratório, quando haverá necessidade de maior gasto energético.

O decréscimo no conteúdo de gordura celomática ao longo dos estádios do ciclo reprodutivo sugere provável utilização das reservas graxas no processo de amadurecimento das gônadas e/ou migração reprodutiva (Wootton, 1995). Comportamento semelhante foi observado em L. piau (Tavares, 1986) e C. lepidura (Andrade, 1990).

Os valores mensais do IGC mais elevados foram obtidos nos meses de março/1998 $(5,86 \pm 1,37)$ e maio $(5,20 \pm 1,37)$, correspondentes ao período que antecedeu o início da maturação gonadal, observada pela captura de exemplares em repouso. $\mathrm{O}$ valor mensal mais baixo foi obtido no mês de setembro/1998 $(0,29 \pm 0,29)$, justamente quando ocorreu pico do RGS e os indivíduos se apresentaram em maturação avançada, aptos à reprodução (Tabela 1).

Em fêmeas de A. bimaculatus, Barreto et al. (1998) e Bazzoli et al. (1998) observaram níveis elevados de gordura celomática antes do período reprodutivo, relatando intenso consumo destas reservas graxas durante a maturação gonadal.

Houve correlação significativa e negativa entre IGC e RGS $(\mathrm{r}=-0,46 ; \mathrm{P}<0,001 ; \mathrm{n}=143)$. Portanto, para L. copelandii, o teor de gordura celomática talvez pos- 
sa ser utilizado como um indicador auxiliar do período reprodutivo e, provavelmente, está associado à demanda energética dos indivíduos ao longo deste.

Para Nikolsky (1963), o conteúdo de gordura de um peixe pode quantificar o bem-estar do indivíduo, e suas variações sazonais estão intimamente ligadas à alimentação e reprodução da espécie. Segundo Junk (1985), citado por Andrade (1990), espécies com sazonalidade pronunciada em relação ao acúmulo de gordura são geralmente de desova total e realizam migrações reprodutivas e/ou tróficas, como é o caso de L. copelandii. O conteúdo de gordura é diretamente proporcional ao comprimento do curso de migração e a velocidade da corrente do rio que o indivíduo tem que transpor (Nikolski, 1963).

$\mathrm{O}$ índice de gordura celomática foi correlacionado significativamente com o fator de condição total $(\mathrm{r}=0,20$; $\mathrm{P}=0,01 ; \mathrm{n}=143)$, e somático $(\mathrm{r}=0,37 ; \mathrm{P}<0,001$; $\mathrm{n}=143$ ), pois o peixe só vai acumular gordura após ter suprida suas necessidades básicas de mantença.

Para Godinho (1994), o acúmulo de gordura celomática está relacionado à quantidade de alimento ingerida e à sua disponibilidade. Porém, neste trabalho, foi observado para $L$. copelandii correlação negativa significativa entre IGC e IRE $(r=-0,26 ; \mathrm{P}<0,001$; $\mathrm{n}=143$ ). Isto reflete um acúmulo de gordura previamente ao processo de migração reprodutiva, pois, no período de reprodução, observou-se um enchimento menor do estomago em função do aumento gonadal.

\section{Conclusões}

1. A diferença entre o fator de condição total (K1) e somático (K2) indica o início do período reprodutivo para Leporinus copelandii no local de coleta, e o fator de condição somático é o melhor indicador da condição corpórea das fêmeas.

2. Verifica-se possível participação do fígado no processo de maturação ovocitária, provavelmente devido à síntese e secreção de substâncias hepáticas que participam da vitelogênese.

3. As fêmeas apresentam-se em melhores condições alimentares após o período reprodutivo, porém não interrompem sua atividade alimentar ao longo do ano na região do baixo Rio Paraíba do Sul.

4. Verifica-se sazonalidade pronunciada em relação ao acúmulo de gordura celomática durante o processo de maturação gonadal de fêmeas de Leporinus copelandii, o que indica a preparação da espécie para o desenvolvimento gonadal e/ou migração reprodutiva.

\section{Referências}

AGOSTINHO, A.A.; BARBIERI, G.; VERANI, J.R.; HAHN, N.S. Variação do fator de condição e do índice hepatossomático e suas relações com o ciclo reprodutivo em Rhinelepis aspera (Agassis, 1829) (Osteichthyes, Loricariidae) no Rio Paranapanema, Porecatu. Ciência e Cultura, v.42, p.711-714, 1990.

ANDRADE, D.R. Biologia reprodutiva da manjuba Curimatella lepidura (Eigenmann \& Eigenmann, 1889) (Pisces, Curimatidae) da Represa de Três Marias, Rio São Francisco, MG. 1990. 217p. Tese (Doutorado) - Universidade Federal de Minas Gerais, Belo Horizonte.

BARBIERI, G. Biologia de Astyanax scabripinnis paranae (Characiformes, Characidae) do Ribeirão do Fazzari. São Carlos. Estado de São Paulo. II. Aspectos quantitativos da reprodução. Revista Brasileira de Biologia, v.52, p.589-596, 1992.

BARBIERI, G.; SANTOS, E.P. Análise comparativa do crescimento e de aspectos reprodutivos da piava, Leporinus friderici (Bloch, 1794) (Osteichthyes, Anostomidae) da Represa do Lobo e do Rio Mogi-Guaçu, Estado de São Paulo. Ciência e Cultura, v.40, p.693-697, 1988.

BARBIERI, G.; SANTOS, M.V.R. dos; SANTOS, J.M. dos. Época de reprodução e relação peso/comprimento de duas espécies de Astyanax (Pisces, Characidae). Pesquisa Agropecuária Brasileira, v.17, p.1057-1065, 1982.

BARBIERI, M.C. Contribuição ao estudo da biologia de Gymnotus carapo (Linnaeus, 1785) na Represa do Lobo, Estado de São Paulo (Pisces, Ostariophysi, Gymnotidae). 1981 220p. Tese (Doutorado) - Universidade Federal de São Carlos, São Carlos.

BARRETO, B.P.; RATTON, T.F.; RICARDO, M.C.P.; ALVES, C.B.M.; VONO, V.; VIEIRA, F.; RIZZO, E.; BAZZOLI, N. Biologia reprodutiva do lambari Astyanax bimaculatus (Pisces, Characidae) no Rio do Carmo, Bacia do Rio Grande, São Paulo. Bios, v.6, p.121130, 1998.

BAZZOLI, N. Biologia reprodutiva do peixe-cachorro Acestrorhynchus lacustris (Reinhardt, 1874) (Characidae, Acestrorhynchinae) da Represa de Três Marias, Rio São Francisco, MG. 1985. 149p. Dissertação (Mestrado) - Universidade Federal de Minas Gerais, Belo Horizonte.

BAZZOLI, N.; MESQUITA, T.L.; SANTOS, G.B.; RIZZO, E. Análise comparativa da reprodução de Astyanax bimaculatus (Pisces, Characidae) nos Reservatórios de Furnas, Marimbondo e Itumbiara. Bios, v.6, p.99-112, 1998.

BAZZOLI, N.; RIZZO, E.; SANTOS, J.E. dos; SATO, Y. Dinâmica da ovogênese de peixes forrageiros da Represa de Três Marias, Minas Gerais: estudo histológico e histoquímico. Bios, v.4, p.5-10, 1996.

DINIZ, C.C. Dinâmica reprodutiva de Leporinus striatus e histologia do ovário de cinco espécies do gênero Leporinus Spix 1829 (Osteychthyes, Characiformes, Anostomidae) na Represa de Camargos, MG. 1997. 69p. Dissertação (Mestrado) Universidade Federal de Lavras, Lavras.

FERREIRA, R.M.A.; GODINHO, H.P. Reproductive biology of the white-piau, Schizodon knerii (Steindachner, 1875) (Anostomidae) from a reservoir in southeast Brasil. European Archives of Biology, v.101, p.331-344, 1990. 
GODINHO, A.L. Biologia reprodutiva da Piaba-Facão, Triportheus guentheri (Characiformes, Characidae) e o manejo hidrológico da Represa de Três Marias. Revista Brasileira de Biologia, v.54, p.515-524, 1994.

JUNK, W. Temporary fat storage on adaptation of some fish species to the water level fluctuations and related environmental changes of the Amazon River. Amazoniana, v.9, p.315-351, 1985.

LE CREN, E.D.The lenght-weight relationship and seasonal cycle in gonad weight and condition in the perch (Perca fluviatilis). Journal of Animal Ecology, v.20, p.201-219, 1951.

LOPES, C. de A.; BENEDITO-CECÍLIO, E.; AGOSTINHO, A.A. The reproductive strategy of Leporinus friderici (Characiformes, Anostomidae). Revista Brasileira de Biologia, v.60, p.255-266, 1999.

NIKOLSKY, G.V. The ecology of fishes. London: Academic Press, 1963. 352p.

PIMENTEL-GOMES, F. Curso de Estatística experimental. 13.ed. Piracicaba: Esalq-USP, 1990. 467p.

SANTOS, G.B. Estudos sobre a biologia de Leporinus piau Fowler, 1941 na Represa de Três Marias (MG) (Pisces,
Ostariophysi, Anostomidae). 1986. 153p. Dissertação (Mestrado) - Universidade Federal de São Carlos, São Carlos.

TAVARES, E.F. Biologia reprodutiva do piau-gordura Leporinus piau Fowler, 1941 (Pisces, Anostomidae) da Represa de Três Marias, Rio São Francisco, MG. 1986. 108p. Dissertação (Mestrado) - Universidade Federal de Minas Gerais, Belo Horizonte.

TAVARES, E.F., GODINHO, H.P. Ciclo reprodutivo do peixe piaugordura (Leporinus piau Fowler, 1941) da Represa de Três Marias, Rio São Francisco. Revista Ceres, v.41, p.28-35, 1994.

TELES, M.E.O.; GODINHO, H.P. Ciclo reprodutivo da Pirambeba Serrasalmus brandtii (Teleostei, Characidae) na Represa de Três Marias, Rio São Francisco. Revista Brasileira de Biologia, v.57, p.177-184, 1997.

VAZZOLER, A.E.A. de M. Biologia da reprodução de peixes teleósteos: teoria e prática. Maringá: NUPELIA, 1996. 169p.

VAZZOLER, A.E.A. de M. Manual de métodos para estudos biológicos de populações de peixes. Reprodução e crescimento. Brasília: CNPq, 1982. 106p.

WOOTON, R.J. Ecology of teleost fishes. London: Chapman \& Hall Pub.,1995. 412p.

Recebido em 8 de abril de 2004 e aprovado em 4 de março de 2005 